\title{
Hedgehog signaling and the retina: insights into the mechanisms controlling the proliferative properties of neural precursors
}

\author{
Morgane Locker, ${ }^{1,3}$ Michalis Agathocleous, ${ }^{2,3}$ Marcos A. Amato, ${ }^{1}$ Karine Parain, ${ }^{1}$ \\ William A. Harris, ${ }^{2}$ and Muriel Perron ${ }^{1,4}$ \\ ${ }^{1}$ Laboratoire "Gènes, Développement et Neurogenèse," Université Paris XI, UMR Centre national de la recherche \\ scientifique (CNRS) 8080, 91405 Orsay, France; ${ }^{2}$ Department of Physiology, Development and Neuroscience, Cambridge \\ University, Cambridge, CB2 3DY, United Kingdom
}

Hedgehog signaling has been linked to cell proliferation in a variety of systems; however, its effects on the cell cycle have not been closely studied. In the vertebrate retina, Hedgehog's effects are controversial, with some reports emphasizing increased proliferation and others pointing to a role in cell cycle exit. Here we demonstrate a novel role for Hedgehog signaling in speeding up the cell cycle in the developing retina by reducing the length of G1 and G2 phases. These fast cycling cells tend to exit the cell cycle early. Conversely, retinal progenitors with blocked Hedgehog signaling cycle more slowly, with longer G1 and G2 phases, and remain in the cell cycle longer. Hedgehog may modulate cell cycle kinetics through activation of the key cell cycle activators cyclin D1, cyclin A2, cyclin B1, and cdc25C. These findings support a role for Hedgehog in regulating the conversion from slow cycling stem cells to fast cycling transient amplifying progenitors that are closer to cell cycle exit.

[Keywords: Hedgehog; retinal stem cells; Xenopus; zebrafish; cell cycle kinetics; cell cycle exit; cyclin; Cdc25C]

Supplemental material is available at http://www.genesdev.org.

Received April 18, 2006; revised version accepted September 5, 2006.

The vertebrate retina is an excellent model for investigating neurogenesis, but the mechanisms controlling progenitor proliferation in this tissue are still poorly understood. At the optic vesicle stage, retinal neuroepithelial cells divide to generate mitotic daughters. Later in the optic cup, at the onset of neuronal differentiation, progenitors produce post-mitotic neurons and glial cells. In frogs and fish, insight into the transition between proliferation and differentiation has been gained through examination of the ciliary marginal zone (CMZ), which is a source of new neuronal cells throughout the life of the animal (Wetts et al. 1989; Dorsky et al. 1995; Perron et al. 1998). The spatial layout of the CMZ recapitulates the temporal order of retinoblast proliferation (Perron et al. 1998). Stem cells, in the most peripheral CMZ, give rise to fast cycling progenitor cells more centrally, which, in turn, produce the differentiated retinal cell types that are incorporated into the retinal layers at the

\footnotetext{
${ }^{3}$ These authors contributed equally to this work.

${ }^{4}$ Corresponding author.

E-MAIL muriel.perron@ibaic.u-psud.fr; FAX 33-1-69-15-68-02.

Article is online at http://www.genesdev.org/cgi/doi/10.1101/gad.391106.
}

central edge of the CMZ. Several signaling pathways including Notch, Wnt, FGF, and Hedgehog (Hh) are active in the CMZ and have been implicated in retinal cell proliferation (Ohnuma et al. 2002a; Kubo et al. 2003; Perron et al. 2003; Moshiri and Reh 2004; Spence et al. 2004; Moshiri et al. 2005; Van Raay et al. 2005; Das et al. 2006; Inoue et al. 2006), but how stem cells become transient amplifying progenitors and finally exit the cell cycle is still a mystery.

The Hh signaling pathway is involved in progenitor proliferation in several parts of the vertebrate brain (Wallace and Raff 1999; Wechsler-Reya and Scott 1999; Lai et al. 2003; Machold et al. 2003; Ahn and Joyner 2005; Palma et al. 2005), and many studies also show this is the case during retinal development (for review, see Amato et al. 2004). However, unraveling $\mathrm{Hh}$ function in the retina has turned out to be complicated, not only because $\mathrm{Hh}$ is used for apparently different functions at different developmental times, but also because published results appear to be discordant.

Several studies in several vertebrate species suggest that $\mathrm{Hh}$ promotes retinal progenitor proliferation (for review, see Roy and Ingham 2002; Amato et al. 2004). Re- 
combinant Sonic Hedgehog (Shh) stimulates proliferation of mouse retinal precursor cells in vitro (Jensen and Wallace 1997; Levine et al. 1997). In agreement with this, overactivation of the Hh cascade in Patched heterozygous mice leads to persistence of proliferative cells at the periphery of the post-embryonic retina, causing this region to resemble the CMZ of lower vertebrates (Moshiri and Reh 2004). Additionally, in post-hatch chicks, intraocular injection of Shh stimulates proliferation in the peripheral retina (Moshiri et al. 2005). Cyclopamine, a pharmacological inhibitor of the $\mathrm{Hh}$ coreceptor Smoothened (Smo), inhibits CMZ proliferation (Moshiri et al. 2005), and conditional ablation of Shh expression in the peripheral mouse retina results in decreased cell cycling and down-regulation of cyclin D1 (Wang et al. 2005). Finally, Hh signaling has recently been shown to be essential for chick retinal regeneration, most likely through stimulation of progenitor cell proliferation (Spence et al. 2004). These data support the idea that $\mathrm{Hh}$ signaling promotes proliferation.

Neumann and colleagues, however, studying retinal development in the zebrafish, reached different conclusions (Stadler et al. 2004). These authors showed that Shh, which is secreted by the first-born retinal ganglion cells (RGC), drives a wave of differentiation, and that interference with Shh signaling blocks this progression (Neumann and Nuesslein-Volhard 2000). Moreover, Syu mutants, which lack Shh, show increased proliferation through inhibition of $p 57$ Kip2 and consequent failure of cell cycle exit (Shkumatava and Neumann 2005). PKA activation, which inhibits Hh signaling, or smo loss of function also leads to defects in retinoblast cell cycle exit in zebrafish (Masai et al. 2005).

Given these discordant results, the question that naturally arises is: What does Hh actually do in the retina? Does it inhibit the cell cycle in zebrafish and activate it in mice and chicks? Or is there a deeper function of Hh that is similar across these species, with contradictory interpretations arising because of differences in the assays used? At which step(s) does Hh affect cell proliferation along the road from stem cell to differentiated cell? The expression patterns of $\mathrm{Hh}$ signaling components in the developing retina and the CMZ of Xenopus laevis (Perron et al. 2003) led us to investigate these questions in this model organism, which is particularly accessible for studying retinal development in vivo. We found that blocking the Hh pathway reduces the number of retinal progenitors that take up bromodeoxyuridine (BrdU), consistent with its proposed function as a mitogen. However, this effect results from slower cell cycle kinetics rather than from an increase in cell cycle withdrawal. Moreover, blocking Hh signaling delays cell cycle exit. Conversely, Hh overexpression accelerates the cell cycle and leads to premature cell cycle exit. Flow cytometry experiments and in vivo analysis of cell cycle kinetics provide evidence that $H$ h stimulates G1/S and G2/M transitions. In situ hybridization experiments revealed that the phosphatase $C d c 25 C$, cyclin B1, cyclin D1, and cyclin $\mathrm{A} 2$ are regulated by $\mathrm{Hh}$ in the Xenopus retina, providing potential targets mediating the effects of $\mathrm{Hh}$ on the cell cycle duration.

By combining different experimental approaches in different animals, and examining the effects of Hh signaling at various stages of retinogenesis, we have been able to show that the distinct phenotypes seen in different species could actually reveal aspects of an underlying role for $\mathrm{Hh}$ in controlling transition from a slow cycling "stem" cell to a faster cycling "transit amplifying" progenitor cell.

\section{Results}

Hh signaling affects retinal progenitor proliferation in the $C M Z$

The expression of Hh pathway genes in the Xenopus CMZ (Perron et al. 2003) suggests that Hh signaling may have a role in proliferation. To test this, two-cell-stage embryos were injected with Shh mRNA, allowed to develop until stage 40-41, and injected with BrdU. As Shh causes patterning defects in the ventral retina (Perron et al. 2003; Lupo et al. 2005), we focused our attention on the dorsal CMZ. Compared with controls, the CMZ of Shh-overexpressing embryos contained a significantly higher number of BrdU-positive cells (Fig. 1A-G).

If $\mathrm{Hh}$ promotes proliferation, interfering with this pathway should inhibit BrdU incorporation in the CMZ. Incubation of Xenopus embryos with cyclopamine, which blocks the Smo receptor (Cooper et al. 1998; Incardona et al. 1998), strongly represses the expression of Hh transcriptional targets X-Patched-1 and Gli1, consistent with its ability to inhibit Hh signaling to a significant degree (Fig. 1H,I; Perron et al. 2003). As previously described (Perron et al. 2003), cyclopamine-treated stage 40 embryos presented typical patterning defects resulting from reduced Hh signaling; that is, mild cyclopic phenotypes, reduced eye size, and impaired RPE differentiation. They also showed a reduction in the number of BrdU-labeled cells in the CMZ (Fig. 1J-P). To confirm this result, both blastomeres of two-cell-stage embryos were injected with anti-Smo morpholino (Mo) oligonucleotides or control Mo. Injected embryos displayed eye defects similar to those seen in cyclopamine-treated embryos (data not shown). The specific blockade of the Hh cascade by anti-Smo Mo was further assessed by the observation of decreased X-Patched-1 expression (Fig. 1Q,R). Similar to cyclopamine, anti-Smo Mo significantly reduced BrdU incorporation in the CMZ (Fig. 1S-Y).

\section{Hh signaling regulates cell cycle kinetics in retinal precursor cells}

In order to understand how Hh works to control proliferation, it is necessary to establish a system in which the cell cycle of proliferating retinal cells can be investigated. At earlier optic vesicle stages of retinal development, most or all of the cells are proliferating, as indi- 
Locker et al.

Figure 1. Hh signaling controls precursor cell proliferation in the CMZ. $(A-F, J-O, S-X)$ BrdU incorporation assays (2-h pulse) at stage 40/41 following Shh RNA injection, cyclopamine treatment, or anti-Smo Mo injection, as indicated. $(E, F, N, O, W, X)$ High magnifications of the $\mathrm{CMZ}$ (delineated with doted lines). (L) Lens; (CR) central retina. Bars: $A-D, J-M, S-V, 80$ $\mu \mathrm{m}, E, F, N, O, W, X, 25 \mu \mathrm{m}$. $(H, I, Q, R)$ In situ hybridization showing decreased $X$-Ptc1 staining in cyclopamine-treated and Smo Mo-injected embryos compared with control ones. Bar, $300 \mu \mathrm{m} .(G, P, Y)$ Quantitative analysis of BrdU incorporation in Shhoverexpressing retinas and following Hh inhibition with cyclopamine or Smo Mo. Values represent the number of BrdU-positive cells in the CMZ per section and are given as mean \pm SEM. $\left(^{\star \star \star}\right) p<0.001$; $\left({ }^{\star \star}\right) p<0.01$ (Student's $t$-test).
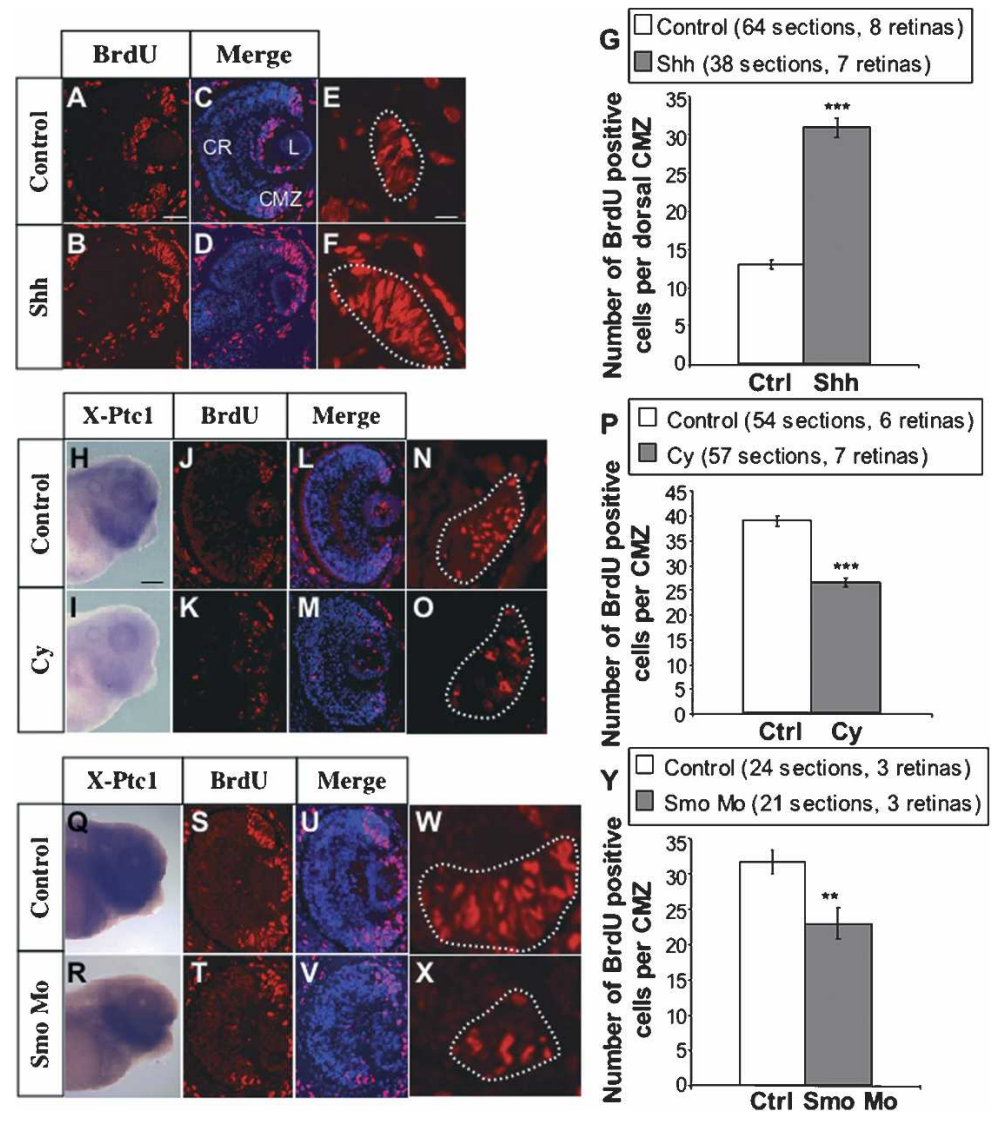

cated by the ubiquitous uptake of BrdU, following a 3-h BrdU pulse at stage 25 (Fig. 2A,B). We therefore considered this as a source of cycling progenitors that could be accessible to flow cytometric analysis of the cell cycle. Since the onset of Hh expression within the retina appears after stage 25 (Perron et al. 2003), we first examined whether retinal precursors at this stage respond to the extraocular source of Hh (Ekker et al. 1995). We found that the Hh targets X-Ptc-1 and Gli1 are expressed in the retinal neuroepithelium, suggesting that the pathway is active within the neural retina at this stage (Supplementary Fig. S1). We then injected cyclopamine-treated embryos with BrdU at stage 25 and fixed retinal cells after $1.5 \mathrm{~h}$. The proportion of BrdU-positive cells decreased from $62 \%$ in controls to $48 \%$ in cyclopamine-treated retinas (Fig. 2C-E).

In a population of asynchronous cycling cells, the fraction of cells in a given phase of the cell cycle is proportional to the length of that phase, relative to the total length of the cell cycle (Nowakowski et al. 1989). Thus, changes in BrdU incorporation do not necessarily reflect a change in the proportion of proliferating cells. They may instead be caused by alterations in the relative lengths of the different phases of the cell cycle. To distinguish between these two possibilities, we first evaluated the mitotic index in the retinal neuroepithelium of stage 24-25 embryos, using the mitotic marker phosphohistone H3 (P-H3). We found a significantly higher percentage of mitotic cells per section $(5.49 \%$ vs. $2.07 \%)$ in
Shh-overexpressing optic vesicles, suggesting that Hh increases the proportion of the cell cycle occupied by the M phase (Fig. 2F-J). A similar in vivo experiment was performed following cyclopamine treatment. However, the high variability in the number of P-H3-labeled cells per retinal section prevented us from observing a clear difference compared with controls. In order to overcome statistical issues that come from counting sections on slides, we turned to flow cytometry, which allowed us to count a much higher number of cells per experiment. We found a reduction of $\sim 20 \%$ in M-phase-positive cells in retinas from cyclopamine-treated embryos compared with controls (Fig. 2K-P). Thus, assuming that at stage 25 all cells are cycling, the modifications observed in the percentage of BrdU and P-H3-labeled cells suggest that Hh modifies cell cycle kinetics, either by extending the duration of both $\mathrm{S}$ and $\mathrm{M}$ phases or by reducing the duration of G1 or G2.

\section{Hh inhibition lengthens G1 and G2 phases of the cell cycle}

To analyze the distribution of cell cycle phases, the DNA content of retinal cells was measured. At stage 25, when almost all cells are still proliferating, cyclopamine treatment caused a reproducible accumulation of retinal precursors in G1 (41\% vs. $36 \%$ in controls). This effect was more obvious at stage 30 , where a corresponding decrease in the S-phase proportion could also be observed 


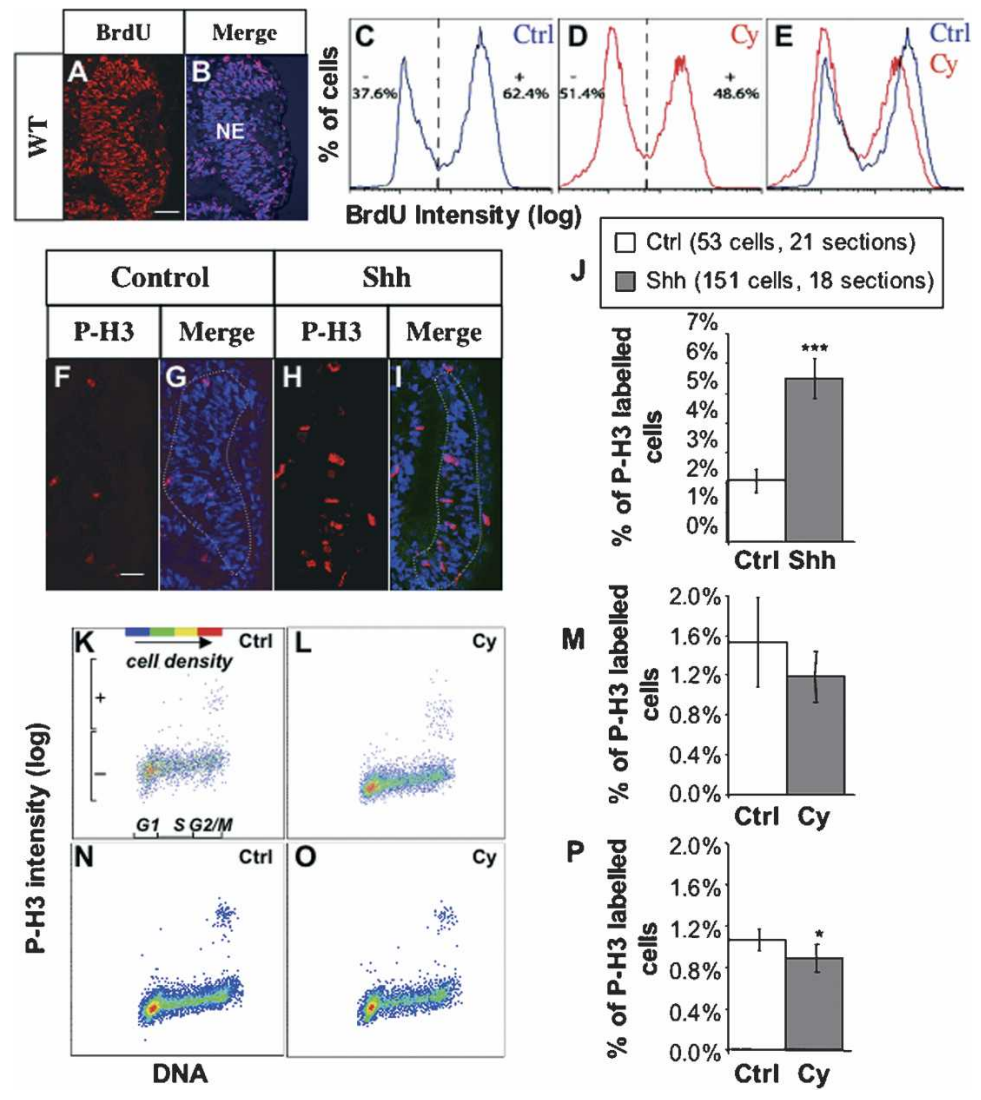

Figure 2. Hh signaling affects cell cycle kinetics of retinal precursors. $(A, B)$ Photographs of retinal neuroepithelium (NE) sections from stage 25 embryos injected with BrdU for $3 \mathrm{~h}$ before fixation. Bar, $50 \mu \mathrm{m}$. $(C-E)$ Flow cytometric analysis of BrdU incorporation (1.5-h BrdU pulse), in retinal neuroepithelium from stage 25 control $(n=13,187$ cells $)$ or cyclopamine-treated $(n=23,459$ cells) embryos; $p<0.001$ (binomial test). ( $F-I)$ Retinal sections from stage 25 embryos injected with GFP RNA or GFP plus Shh RNAs, immunostained with antibodies against GFP (not shown) and phospho-Histone H3 (P$\mathrm{H} 3)$. Dotted lines delineate neural retinas. Note that Shh-overexpressing optic vesicles are substantially thinner compared with control ones, probably due to early morphogenetic defects. Bar, $30 \mu \mathrm{m}$. (J) Quantification of the proportion of mitotic cells in the neuroepithelium of injected embryos. The area of each section was measured. The mean number of cells per section was then inferred from the counting of Hoechst-positive nuclei in a restricted area of each section, and was used to calculate the percentage of P-H3-labeled cells per section. Values are given as mean \pm SEM. $\left(^{\star \star \star}\right) p<0.001$ (Student's $t$-test). $(K-P)$ Bivariate flow cytometric analysis of P-H3/DNA-stained retinal cells from control or cyclopamine-treated embryos at stage $25(K-M)$ and stage 30 $(N-P) .(M)$ At stage 25, the proportion of P-H3-labeled cells decreases from $1.55 \%(K, 2845$ cells $)$ in control to $1.20 \%$ in cyclopamine-treated embryos $(L, 6671$ cells $)$, but this is not significant presumably because of low cell numbers. $(P)$ At stage 30, the percentage of mitotic cells is significantly reduced from $1.07 \%$ in control $(N$, 32,476 cells) to $0.89 \%$ in cyclopamine-treated embryos $(O, 18,763$ cells $)$. Error bars represent $95 \%$ confidence intervals. $\left(^{*}\right) p<0.05$ (one-tail binomial test).
(Fig. 3A,B). These results have the same limitations as the above BrdU and $\mathrm{P}-\mathrm{H} 3$ results-namely, that they are informative only about the duration of cell cycle phases relative to each other. In order to follow more closely the progression of retinal precursors through the cell cycle, we adapted the BrdU/DNA bivariate flow cytometric technique (Dolbeare et al. 1983) to the retina. BrdU is injected in stage 25 embryos; retinas are then fixed and dissociated and analyzed for both BrdU incorporation and DNA content. With no time interval between BrdU injection and fixation, only S-phase cells should be BrdUpositive. An interval, however, allows BrdU-positive cells to progress to subsequent phases of the cell cycle, and hence the proportions of the BrdU-labeled cells in the different cell cycle phases can give an indication of the rate of cell cycle traversal. In the following experiments, we allowed a 1.5 -h injection-fixation interval. We separated the cells in six different groups, according to their cell cycle phase and their level of BrdU incorporation (Fig. 3C,D; see Material and Methods). The proportion of cells in each group differed significantly between cyclopamine-treated and control embryos for five of the six compartments (Fig. 3E). Based on the proportions of cells in each group, we determined cell cycle progression parameters (see Material and Methods). The proportion of $\mathrm{G} 2 / \mathrm{M}$ cells that remain BrdU-negative $\left(\pi G 2 \mathrm{M}^{-}\right)$is proportional to the length of $\mathrm{G} 2 / \mathrm{M}$. We found that cyclopamine increases this measure, showing that cells devoid of Hh signaling spend a longer time in G2/M phase (28\% vs. 16\%) (Fig. 3F). Since the proportion of M-phase cells decreases with cyclopamine treatment, these results show that the G2 phase must be longer when the Hh pathway is inhibited. The rate of G1-S transition $(\Delta G 1)$, derived from the proportion of G1 cells entering $S$ phase in a small time window, is reduced by $40 \%$ after cyclopamine treatment, indicating that $\mathrm{G} 1$ is also prolonged (Fig. 3F). In contrast, the rate of exit from $S$ phase $(\Delta S$ and $\pi+G 2 M)$ is similar in control and cyclopamine retinas, suggesting that $S$-phase duration is unaffected by Hh signaling (Fig. 3F). Importantly, cyclopamine exposure from stage 24 to stage 32 produced similar results (Supplementary Fig. S2), ruling out the possibility that these effects could be secondary to defects in early optic vesicle patterning.

We next wanted to evaluate G2 length using the percentage of labeled mitoses (PLM) paradigm (Fig. 3G; Quastler and Sherman 1959). Stage 32 embryos injected with BrdU were allowed to recover from $15 \mathrm{~min}$ to $3 \mathrm{~h}$ before fixation and stained for BrdU and P-H3 (Fig. 3H-J). As expected from the asynchrony among retinal cells, the percentage of $\mathrm{P}-\mathrm{H} 3 /$ BrdU-labeled nuclei increased sigmoidally with increasing BrdU exposure times, before flattening out at $100 \%$. The percentage of P-H3/BrdUpositive cells was consistently lower in cyclopamine- 
Locker et al.

Figure 3. Hh signaling inhibition extends G1- and G2-phase duration in vivo. $(A, B)$ Flow cytometric analysis showing cell phase distribution of retinal precursors from control or cyclopamine-treated embryos at stage 25 or 30 . The bar chart indicates the percentages of cells in each phase of the cell cycle at stage 30. $(C-F)$ Bivariate flow cytometric analysis of BrdU/DNA-stained retinal cells from stage 25 control $(C, 13,187$ cells) or cyclopamine-treated $(D$, 23,459 cells) embryos (1.5-h BrdU pulse). (E,F) Graphs showing proportions of cells in each compartment $(E)$ and derived cell cycle parameters values $(F)$. Error bars represent $95 \%$ confidence intervals. $\left(^{\star \star \star}\right) p<0.001$ (binomial test). $(G-K)$ G2 length evaluation in vivo. $(G)$ Schematic representation of the experiment. During the BrdU injection interval, cells that go through S phase are BrdU-labeled. Following fixation, mitotic cells $\left(\mathrm{P}-\mathrm{H}^{+}\right)$that are $\mathrm{BrdU}^{+}$ have progressed from $S$ to $M$ phase. By inference, $M$ cells found as $\mathrm{BrdU}^{-}$were already in $\mathrm{G} 2$ or M phase during the BrdU incorporation time. Therefore, the percentage of $\mathrm{P}-\mathrm{H}^{+} / \mathrm{BrdU}^{-}$cells is proportional to G2 duration. $(H-J)$ Example of double immunostaining against $\mathrm{BrdU}$ and $\mathrm{P}-\mathrm{H} 3$ on stage 32 retinal sections, following a $1.25 \mathrm{~h}$ BrdU pulse. The arrow and arrowhead point to a $\mathrm{P}-\mathrm{H}^{+} / \mathrm{BrdU}^{-}$and to a $\mathrm{P}-\mathrm{H}^{+} / \mathrm{BrdU}^{+}$cell, respectively. (L) Lens; (NR) neural retina. Bar, $30 \mu \mathrm{m}$. $(K)$ Quantitation of the percentage of P-H3/BrdU colabeled nuclei along with increasing BrdU exposure times. Values are given as mean \pm standard error.
A
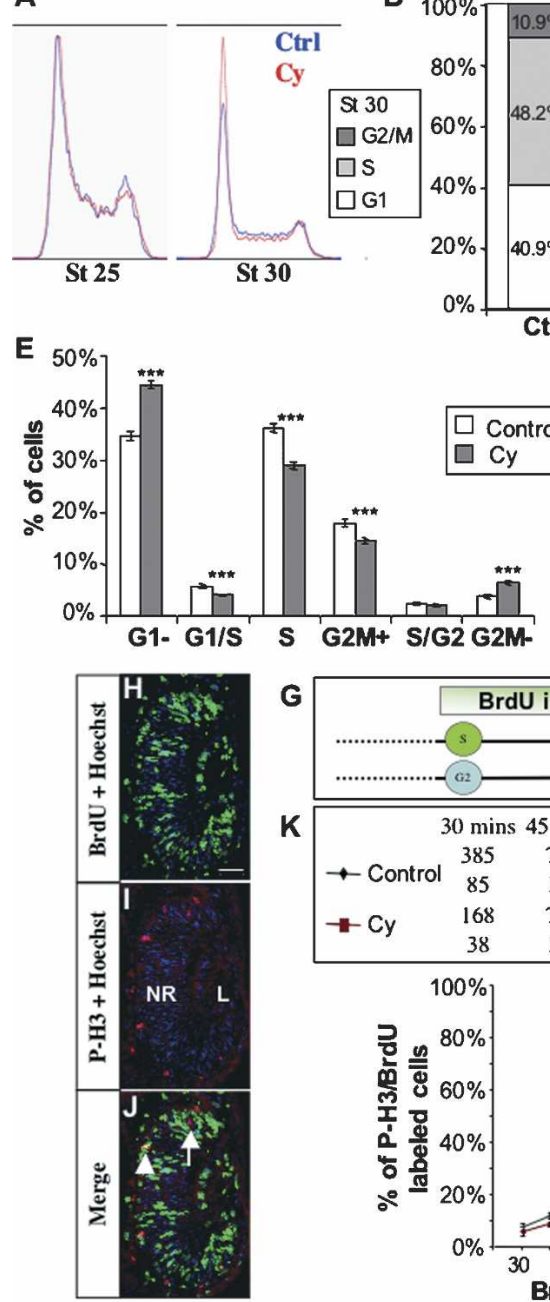

C

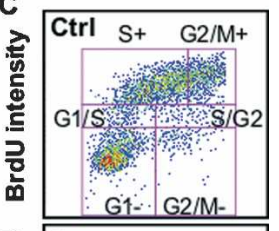

D

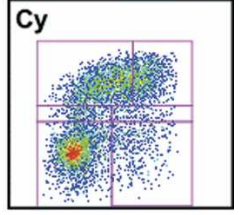

DNA F
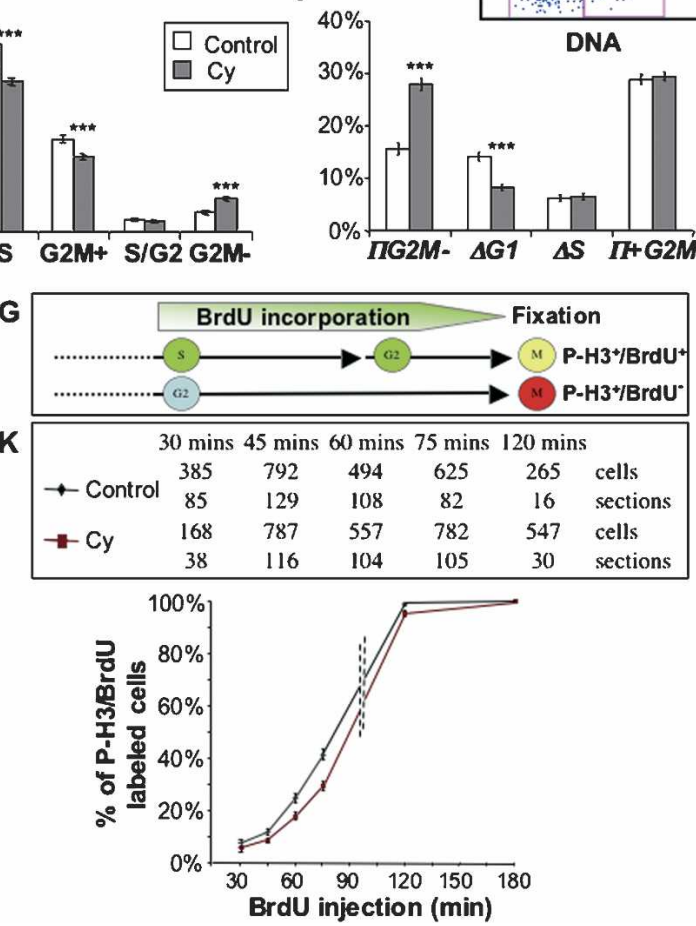

treated retinas compared with controls (Fig. $3 \mathrm{~K}$ ), indicating a longer G2-phase duration. Similar results were obtained in the CMZ of stage 38 embryos following a 2-h BrdU pulse (Supplementary Fig. S3A).

\section{Hh pathway activation accelerates G1 and G2}

If cyclopamine lengthens G1 and G2, activation of the Hh pathway may specifically accelerate these phases. Analysis of DNA content showed an increase in the proportion of cells in S phase, and a decrease in G1 in Hhoverexpressing retinas (Fig. 4A,B), consistent with this hypothesis. However, as mentioned above, Hh overexpression leads to patterning defects in the ventral retina. In order to minimize this potential complication, we took advantage of an inducible Gli1 construct, VP16Gli1zfGR (Takabatake et al. 2002). RNA encoding VP16Gli1zfGR was injected in two-cell-stage embryos, and its activity was induced from stage 25 to stage 32 by addition of dexamethasone (DEX). BrdU was injected at stage 32 , and the retinas were collected $2.5 \mathrm{~h}$ later for flow cytometric analysis. Overall, BrdU incorporation was significantly increased compared with controls (Fig. $4 \mathrm{C})$. Using BrdU/DNA bivariate analysis as above, we found that in contrast to the loss-of-function experiments, stimulation of Gli target genes resulted in a shortening of both G1 and G2 phases (Fig. 4D-H). The rate of entry into $S$ phase $(\Delta G 1)$ significantly increased from $14.9 \%$ in the GFP controls to $46.8 \%$ in VP16Gli1zfGR-induced retinas, while the proportion of G2/M BrdU-negative cells decreased from $36.4 \%$ to $24.7 \%$ (Fig. $4 \mathrm{H}$ ). As in the cyclopamine experiments, the duration of $S$ phase (see $\Delta S$ and $\pi+G 2 M$ ) was not altered (Fig. $4 \mathrm{H})$. Therefore, we conclude that $\mathrm{Hh}$ signaling is sufficient to accelerate G1 and G2 phases.

\section{Cell cycle components are affected by Hh signaling}

To examine whether Hh could achieve its effects on the cell cycle by changing core cell cycle component expression, we focused on the main cyclins expressed in the retina: cyclins A2, B1, and D1 (Ohnuma et al. 2002a; Vernon and Philpott 2003). The expression of these genes was examined by in situ hybridization in control and 


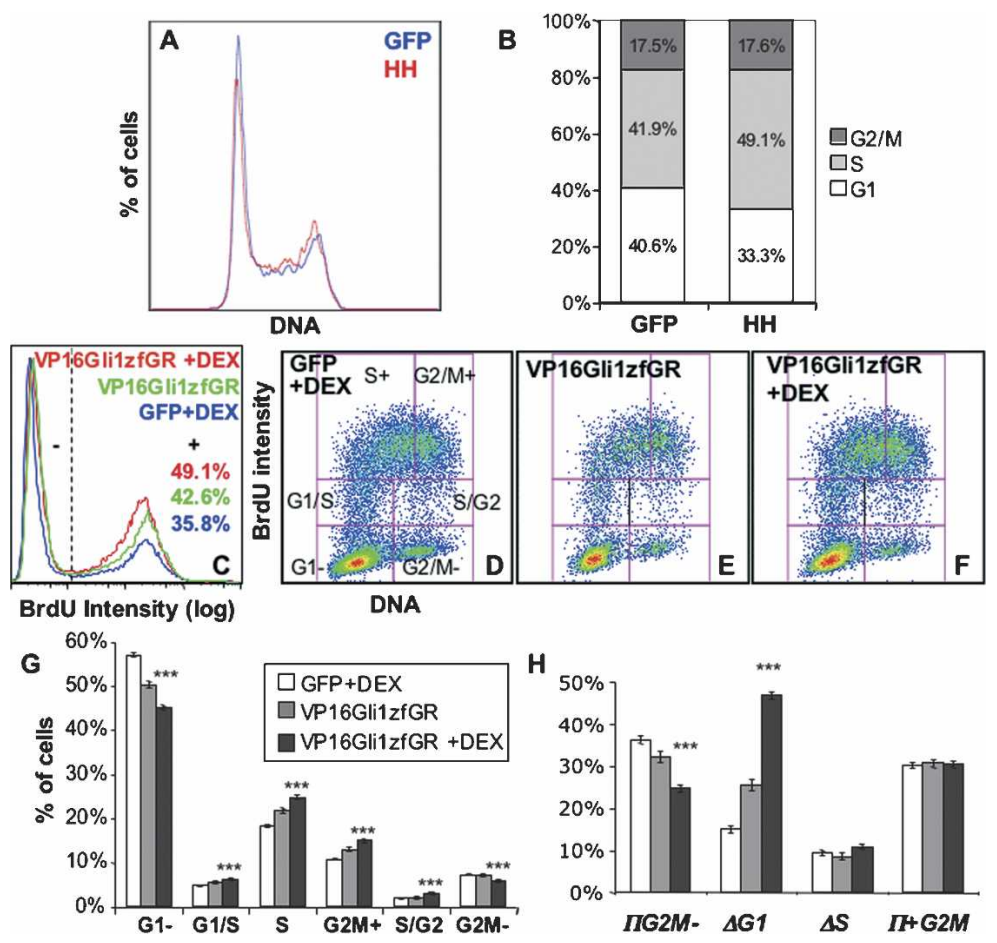

Figure 4. Activation of the Hh pathway shortens G1 and $\mathrm{G} 2$ phases. $(A, B)$ Flow cytometric analysis showing cell phase distribution of retinal precursors from stage 25 control or Hh-overexpressing embryos. The bar chart indicates the percentages of cells in each phase of the cell cycle. $(C-H)$ Bivariate flow cytometric analysis of BrdU/DNA-stained retinal cells following injection with GFP or VP16Gli1zfGR RNA. DEX was added to the embryo medium at stage $24 / 25$, and embryos were injected with BrdU (2.5-h pulse) at stage 32. (C) Level of BrdU incorporation following DEX-dependent activation of the chimeric Glil protein $(37,232$ cells) compared with controls (uninduced VP16Gli1zfGR-injected embryos, 22,078 cells; and DEX-treated GFP-injected embryos, 55,459 cells). (D-F) BrdU versus DNA staining scatterplots for the three conditions. $(G, H)$ Graphs showing proportions of cells in each compartment $(G)$ and derived cell cycle parameters values $(H)$. Of note, the uninduced VP16Gli1zfGR group exhibits a phenotype intermediate between the induced and the GFP groups, indicating a small leakage of the construct without hormone induction. Error bars represent $95 \%$ confidence intervals. $\left({ }^{\star \star \star}\right) p<0.001$ (binomial test).

cyclopamine-treated Xenopus embryos at stages 30/32 and 38/39. Cyclin D1 expression was slightly reduced in the retinas of cyclopamine-treated embryos (Fig. 5I-L), in accordance with previous findings (Kenney and Rowitch 2000; Wang et al. 2005). We also found that cyclin A2 and cyclin $B 1$ expression was significantly repressed upon cyclopamine treatment (Fig. 5A-H). The decrease in cyclin A2 expression was minor, whereas the decrease in cyclin B1 expression was much stronger. Finally, we found that the expression of the Cdc25C phosphatase, which activates G2/M transition, was strongly inhibited in the retina of cyclopamine-treated embryos (Fig. 5MP). These results are not likely to be secondary to changes in the size of the CMZ as expression of the CMZ marker XRX1 (Perron et al. 1998) did not significantly differ between control and cyclopamine-treated retinas (Fig. 5Q,R).

To investigate whether opposite effects on cell cycle components could be observed following activation of the Hh pathway, we injected embryos with RNA encod-

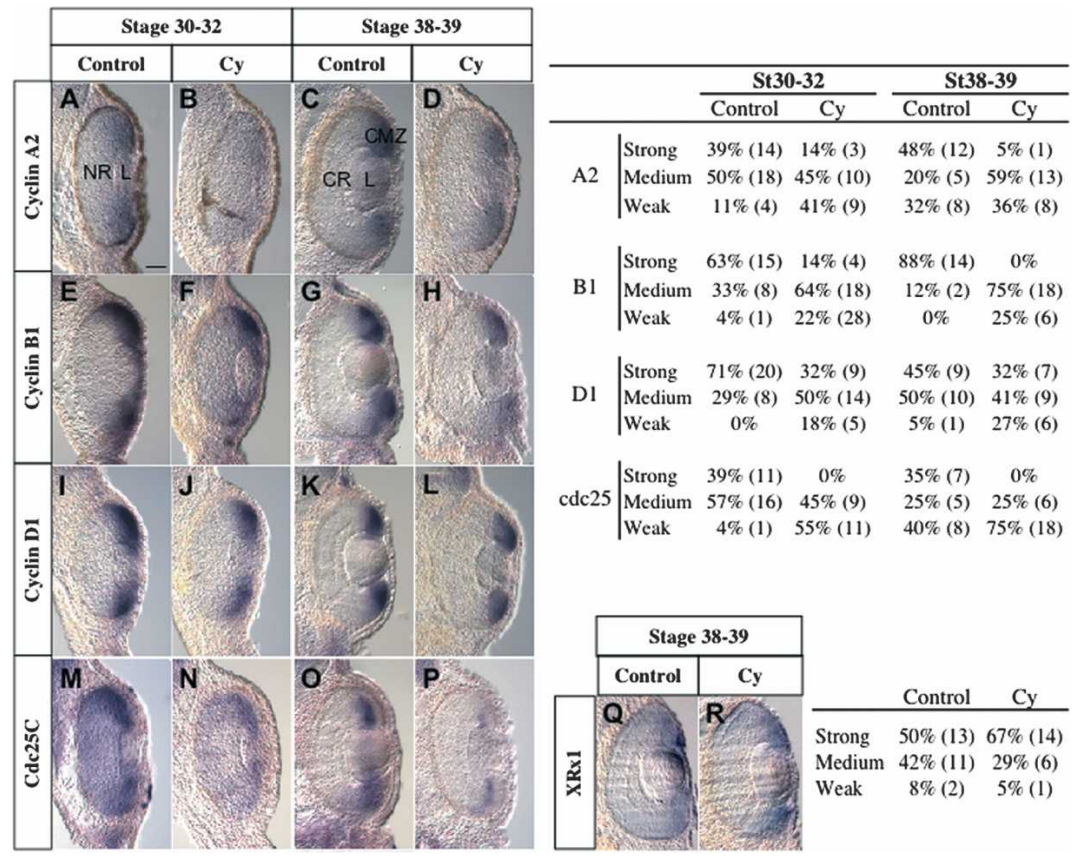

Figure 5. Blocking the Hh cascade decreases the expression of cyclin A2, cyclin B1, cyclin $D 1$, and $C d c 25 C$ phosphatase. Analysis of cyclin A2, cyclin B1, cyclin D1,Cdc25C, and $X R \times 1$ expression on retinal sections after whole-mount in situ hybridization performed on cyclopamine-treated or control embryos at stages $30 / 32$ and $38 / 39$, as indicated. Tables indicate the percentage and number of CMZs exhibiting a weak, medium, or strong staining for each probe and condition, following blind analysis. As in the strongest cyclopic phenotypes the morphology of the ventral part of the retina is quite affected, we also performed the analysis considering only the dorsal part of the retina and obtained similar results. (L) Lens; (NR) neural retina; (CR) central retina. Bar, $40 \mu \mathrm{m}$. 
Locker et al.

ing VP16Gli1zfGR (Supplementary Fig. S4). DEX was added for $8 \mathrm{~h}$ at stage 23. As expected, activation of the construct led to a significant increase in X-Ptc1 expression compared with the uninduced embryos. As the VP16Gli1zfGR construct is slightly leaky (Fig. 4H), uninduced VP16Gli1zfGR embryos exhibited an intermediate intensity of $X$-Ptc1 staining compared with control embryos treated with DEX. Increased expression was also detectable for all the cyclins and cdc25 in uninduced VP16Gli1zfGR-injected embryos compared with control ones. We could not detect any further up-regulation for cyclin B1 and cdc25 following the 8-h induction period. Cyclin D1 expression, however, was significantly enhanced, while Cyclin A2 expression was slightly increased. The changes in cell cycle length may thus be due to the influence of $\mathrm{Hh}$ signaling on the expression of cell cycle machinery.

\section{Hh inhibition results in prolonged proliferation during retinal neurogenesis}

The effects of Hh signaling on the expression of cell cycle stimulators and on the rates of cell cycle transition pose the question of whether $\mathrm{Hh}$ is also able to keep cells proliferating for longer during histogenesis by increasing cell cycle re-entry rates. To investigate this, we measured the proportion of BrdU-labeled cells at various stages of retinal neurogenesis in cyclopamine-treated embryos (Fig. 6A-E). At stage 31, when many cells in the central retina are still cycling, cyclopamine treatment significantly reduced the percentage of BrdU-labeled cells in the retina, as expected from slower cell cycle kinetics $(42.12 \% \pm 1.71$ vs. $52.18 \% \pm 2.28$ in control retinas). At later stages, however, a significant increase could be observed upon $\mathrm{Hh}$ signaling inhibition $(55.70 \pm 2.58$ cells per section vs. $49.51 \pm 1.47$, at stage 34 ; and $19.11 \pm 1.39$ cells per section vs. $12.1 \pm 0.98$, at stage 36 ), suggesting that, as neurogenesis progresses, the proportion of proliferating cells declines more slowly in cyclopamine-treated retinas compared with controls (Fig. 6A). Consistent with this, the number of mitotic cells in the central retina was higher in stage 38 cyclopaminetreated embryos compared with control ones (Supplementary Fig. S3C). Interestingly, stage 38 provides a window into several steps of retinogenesis; that is, "late retinogenesis" in the central retina and "early retinogenesis" in the CMZ (Harris and Perron 1998). In cyclopamine-treated stage 38 embryos, while the number of $\mathrm{M}$ -
Figure 6. Hh inhibition delays cell cycle withdrawal of retinal precursors. (A) Proportion of BrdU incorporation (3-h pulse) in cyclopamine-treated retinas relative to controls, at stages 31,34 , and 36 . BrdU incorporation was monitored at stage 31 in a subpopulation of GFP lipofected cells ([***] $\mathrm{p}<0.001$ [binomial test]), while at stages 34 and 36 , direct counting of $\mathrm{BrdU}^{+}$cells in the central retina was performed $\left.\left({ }^{\star \star \star}\right] p<0.001 ;{ }^{*}\right] p<0.05$ [Student's $t$-test]). $(B-E)$ Typical sections from stage 36 control and cyclopamine-treated retinas, immunostained for BrdU. Compared with controls, central retinas from cyclopamine-treated embryos contain a greater number of cycling cells (arrowheads), mainly localized in the ventral region (arrow). $(F-K)$ Birthdating experiments following Hh signaling inhibition. (F) Schematic representation of the experiment. GFP lipofected cells that have exited the cell cycle before the beginning of BrdU injections are $\mathrm{BrdU}^{-}$at stage 41 , whereas cells that have exited the cell cycle at any time during the BrdU incorporation period are $\mathrm{BrdU}^{+}$. $(G)$ Percentage of $\mathrm{BrdU}^{+}$cells among all GFP-transfected cells or among $\mathrm{GFP}^{+} \mathrm{e}$ RGCs. Values are given as mean \pm standard errors. $\left({ }^{\star \star \star}\right) p<0.001$ (binomial test). $(H-K)$ Example of a strong phenotype in cyclopamine-treated embryos with large increase of BrdU-labeled cells in the outer, inner, and RGC layers, compared with controls. $(L)$ TUNEL assay performed on retinal sections from control and cyclopamine-treated embryos at the indicated stages. Values represent the number of apoptotic cells per retinal section and are given as mean \pm SEM. $\left(^{\star \star \star}\right) p<0.001 ;\left(^{\star \star}\right) p<0.01$ (Student's $t$-test). (L) Lens; (CR) central retina. Bar, $40 \mu \mathrm{m}$.
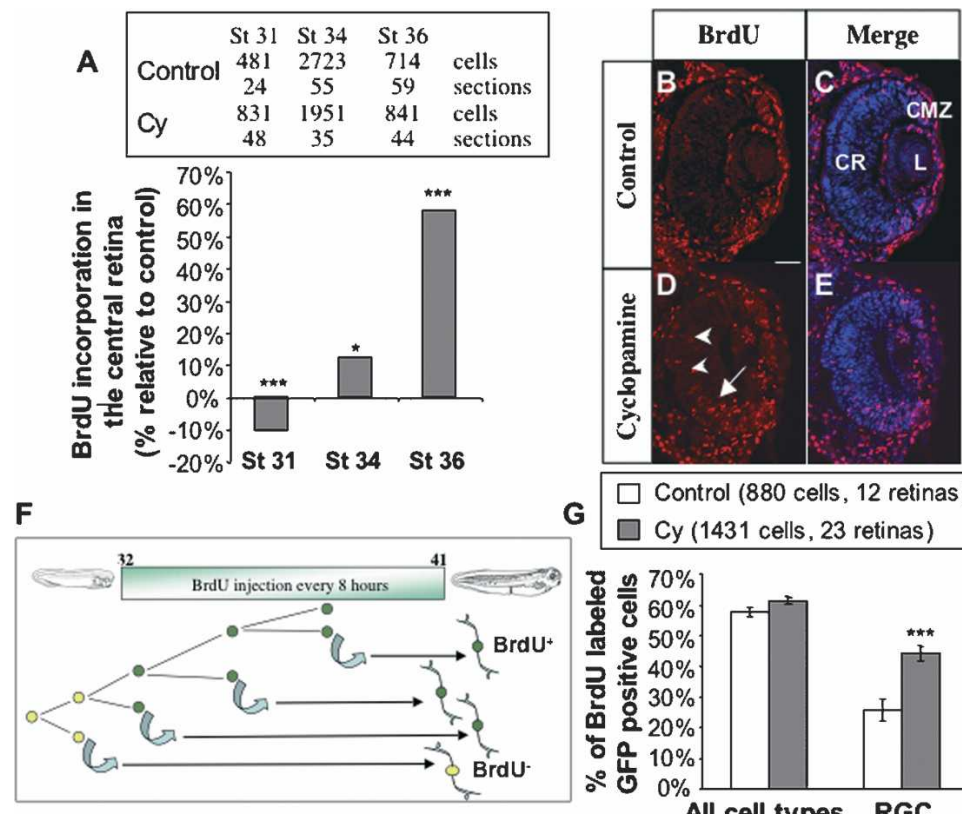

G
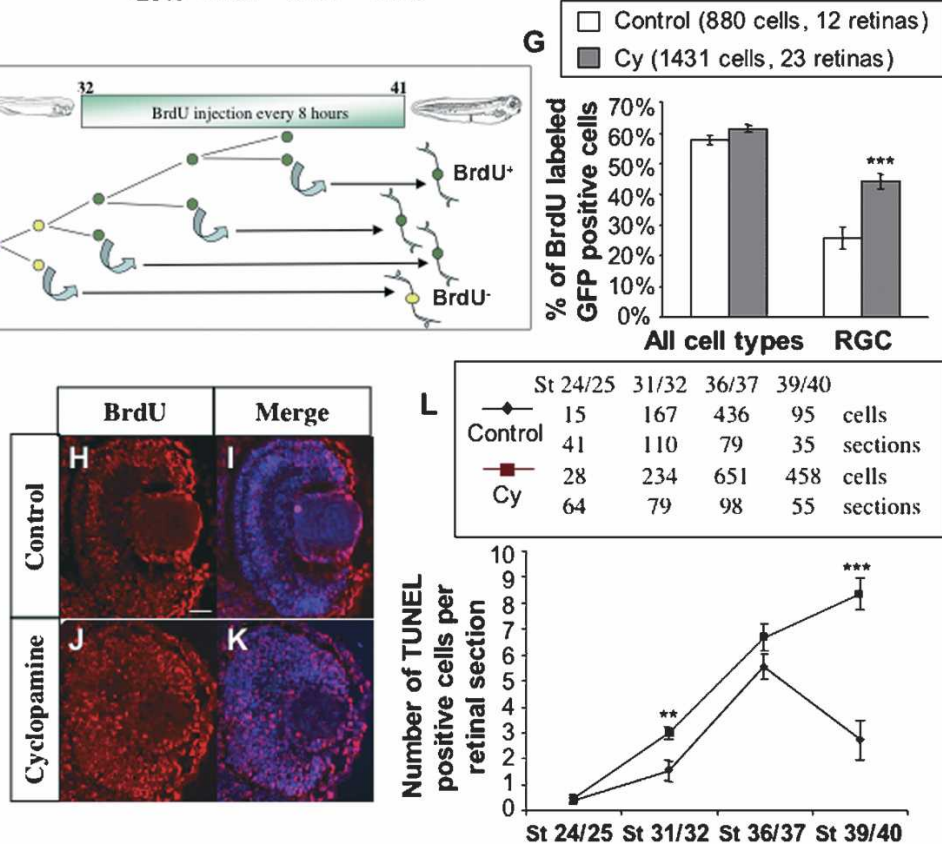
phase cells is increased in the central retina, there is a significant reduction of P-H3-labeled cells in the CMZ, as expected from the altered cell cycle kinetics (Supplementary Fig. S3B).

To examine the timing of cell cycle exit more directly, we performed birthdating experiments that allow the evaluation of the timing of cell cycle exit of each differentiated cell type (Fig. 6F). BrdU was injected at regular intervals so that it would be constantly available from stage 32 to stage 41, and therefore mark all cells born in that period. As a delay in cell cycle exit should be more readily observed for cells normally born before stage 32 , we focused our analysis on RGCs, the first-born cells in the retina (Holt et al. 1988). We found a significant increase in the proportion of BrdU-labeled RGCs in cyclopamine-treated embryos compared with controls (Fig. 6G-K), showing that RGCs from $\mathrm{Hh}$ activity-deficient retinas exit the cell cycle on average later than control RGCs. Therefore, the observed switch in the proportion of BrdU-positive cells during retinogenesis reflects the fact that cell cycle kinetics slow down on the one hand, which tends to decrease the proportion of BrdU-labeled cells, while on the other hand, delayed cell cycle exit results in an increased proportion of proliferating cells.

The smaller eye size of cyclopamine-treated embryos suggests that the prolonged proliferation of retinal progenitors may be overridden by increased cell death, as observed in the zebrafish (Masai et al. 2005; Shkumatava and Neumann 2005). Indeed, we found that retinal cell survival was consistently impaired at stage 39/40 upon Hh inhibition, as inferred from the increased number of TUNEL-positive cells (Fig. 6L). However, the number of apoptotic cells in the retinas of cyclopamine-treated embryos was either indistinguishable or only slightly increased compared with controls, from stage 24 to stage 37. These findings show that Hh signaling may play a role in retinal cell survival after the period of neurogenesis has finished, but exclude a role for apoptosis in our observed proliferation effects during retinogenesis.

\section{Hh overexpression leads to precocious cell cycle exit}

If blocking Hh signaling delays cell cycle withdrawal, Hh activation should accelerate cell cycle exit. To test this idea, we lipofected stage 18 retinas with Bhh-CD2, which encodes a fusion Banded Hh protein linked to the human CD2 surface antigen (Brown et al. 1987). The CD2 domain anchors the fusion protein to the plasma membrane, allowing a cell-autonomous activation of the $\mathrm{Hh}$ pathway in addition to nonautonomous effects. Lipofected embryos were injected with BrdU either at stage 32 or 35 and allowed to recover for $3 \mathrm{~h}$ before fixation (Fig. 7A-C). At stage 32, we found that the proportion of BrdU-positive cells in Hh-overexpressing clones was higher than in controls, consistent with accelerated cell cycle kinetics. By stage 35, however, the proportion of BrdU-labeled cells was significantly lower than controls (Fig. 7D). To test whether this could be related to precocious cell cycle exit, we performed birthdating experiments by injecting BrdU from stage 32 to stage 41 . The
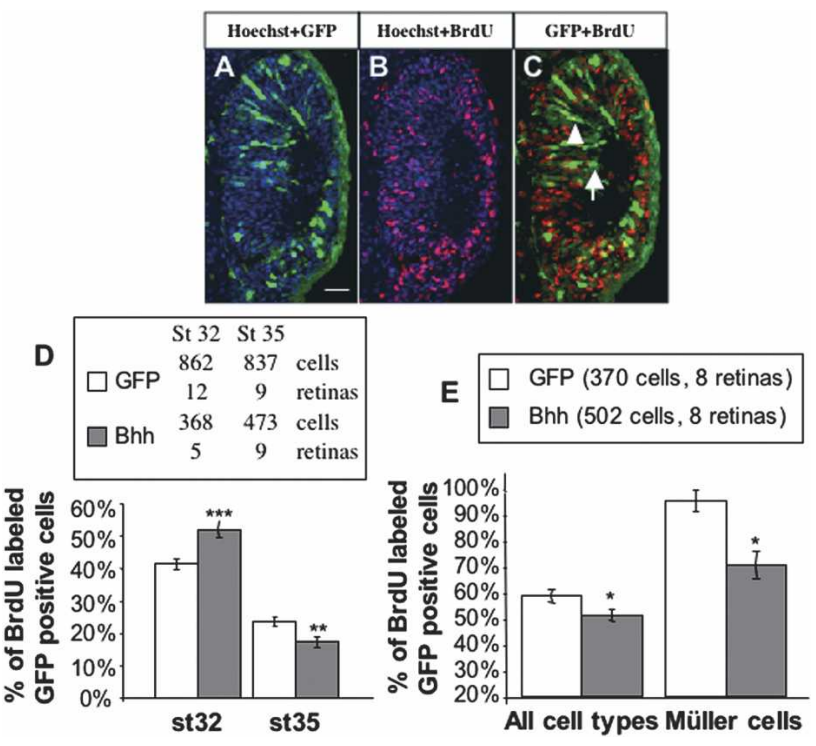

Figure 7. Hh overexpression leads to precocious cell cycle exit. $(A-D)$ Analysis of BrdU incorporation (3-h pulse) in retinal clones at stage 32 or 35 , following Bhh-CD2 lipofection. $(A-C)$ Typical sections of stage 32 retinas immunostained for both GFP and BrdU. The arrow and arrowhead point to a $\mathrm{GFP}^{+} / \mathrm{BrdU}^{-}$ and to $\mathrm{GFP}^{+} / \mathrm{BrdU}^{+}$cell, respectively. $(D)$ Percentage of $\mathrm{BrdU}^{+}$ nuclei among transfected cells. $(E)$ Birthdating experiments following Bhh-CD2 overexpression. Values are given as mean \pm standard errors. $\left.\left(^{\star \star \star}\right) p<0.001 \dot{(}^{(\star \star}\right) p<0.01$ (binomial test). Bar, $20 \mu \mathrm{m}$.

proportion of cells born after stage 32 was significantly reduced in Bhh-CD2 lipofected cells, indicating that Hhoverexpressing cells not only cycle faster, but also exit the cell cycle earlier. This is particularly striking for Müller glia (normally the last cell type born in the retina), as the percentage of Müller cells born before stage 32 increased to $29 \%$ in Bhh-CD2 lipofected retinas compared with $4 \%$ in controls (Fig. 7E). Similar birthdating results were obtained following lipofection and activation of the inducible VP16Gli1zfGR-expressing construct $133 \%$ of BrdU-negative Müller cells in VP16Gli1zfGR clones vs. $13 \%$ in the control; $\chi^{2}$ test, $p=0.001)$.

\section{Hh functions as a regulator of cell cycle kinetics during zebrafish retinogenesis}

Our direct measurements of the effects of Hh signaling on cell cycle exit in Xenopus are in accordance with reports showing that in the zebrafish retina $\mathrm{Hh}$ promotes cell cycle withdrawal. However, our finding that $\mathrm{Hh}$ accelerates the cell cycle of Xenopus retinal progenitors also supports previous work performed in chicks and mammals showing a mitogenic function of the Hh pathway. We therefore thought it was important to investigate whether Hh signaling affects cell cycle kinetics in the zebrafish in the same way as it does in Xenopus. To examine this possibility, we repeated our BrdU/DNA flow cytometric analysis in wild-type zebrafish embryos 
and in embryos mutant for the Hh coreceptor Smo (smu mutants). Embryos were injected with BrdU for $1.5 \mathrm{~h}$ at 26 hours post-fertilization (hpf). At this stage, which just precedes the beginning of retinal neurogenesis, almost all cells are still cycling. Consistent with the Xenopus results, we found that BrdU labeling of retinal precursor cells is decreased from $66 \%$ in wild type to $53 \%$ in smu retinas (Supplementary Fig. S5A). Cell cycle kinetics parameters differed between smu and wild-type retinas in the same way as between cyclopamine-treated and control Xenopus retinas (Supplementary Fig. S5B-E). Both G1 and G2 phases exhibited an increased duration, as assessed by the proportion of BrdU-negative G2/M cells $\left(\pi G 2 M^{-}=6 \%\right.$ in wild type vs. $11 \%$ in smu mutants $)$ and the rate of entry into $S$ phase $(\Delta G 1=36 \%$ in wild type vs. $22 \%$ in smu mutants) (Supplementary Fig. S5E). These results thus highlight a conserved role for $\mathrm{Hh}$ signaling in the control of retinal precursor cell cycle kinetics.

\section{Discussion}

In this study different experimental strategies, including histological assessment of proliferation and flow cytometry, were used with both gain- and loss-of-function approaches to examine the role of $\mathrm{Hh}$ signaling in the cell cycle of retinal cells in two vertebrate species. The results were consistent with a conserved function of $\mathrm{Hh}$ in the control of cell cycle kinetics, through the regulation of G1 and G2 length in retinal precursor cells. Examination of multiple stages of Xenopus retinogenesis revealed additional effects of $\mathrm{Hh}$ on cell cycle exit. Hh-overexpressing fast cycling cells tend to exit the cell cycle early, while slow dividing cells with blocked Hh signaling exhibit delayed cell cycle withdrawal (Fig. 8). Hh signaling regulates the expression of key cell cycle regulators, which may account for these effects.

Most studies of proliferation use static analysis of BrdU incorporation and assume that the percentage of BrdU-positive cells reflects the fraction of actively dividing cells. We aimed for a more dynamic monitoring of the transitions through the cell cycle and by so doing revealed quite a different picture from what had been described before. Hh signaling does, indeed, promote cell cycle progression, consistent with its previously described action as a mitogen (Jensen and Wallace 1997; Levine et al. 1997; Moshiri and Reh 2004; Spence et al. 2004; Moshiri et al. 2005; Wang et al. 2005). However, this is followed by central retinal progenitor depletion due to premature cell cycle exit. Conversely, $\mathrm{Hh}$ inhibition decreases the rate of cell cycling by extending the durations of G1 and G2 and at the same time maintains the proliferative cohort for longer, consistent with results in zebrafish mutants (Masai et al. 2005; Shkumatava and Neumann 2005). Our data may therefore explain many of the apparent differences reported for the role of $\mathrm{Hh}$ in retinal development in different vertebrate species. Hh does not solely direct cell cycle exit, as zebrafish studies had previously suggested, because during early neurogenesis BrdU incorporation in both zebrafish

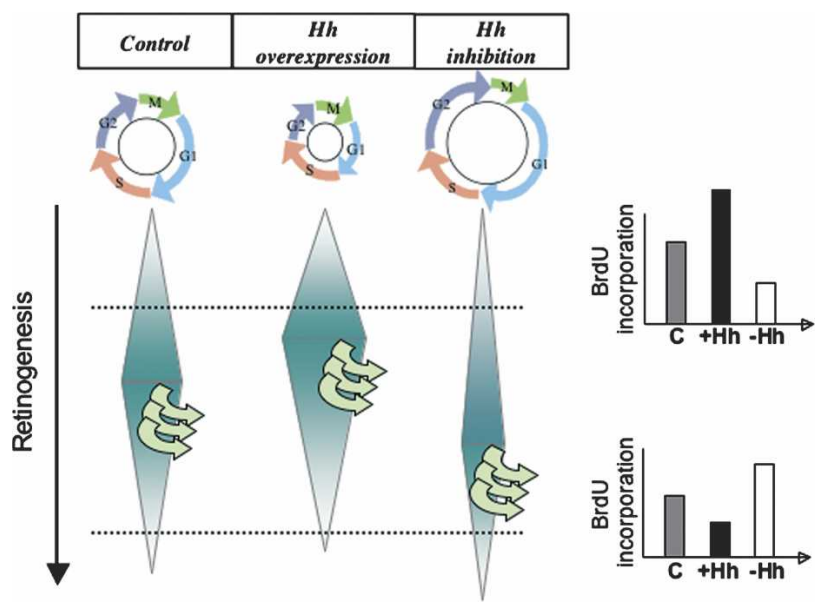

Figure 8. Schema of Hh signaling effects on the retinal proliferative cohort. In the control situation, the pool of proliferative cells (green quadrilaterals) increases during early stages of retinogenesis and then decreases due to cell cycle exit (arrows). Overexpression of Hh leads to an accelerated cell cycle followed by premature cell cycle withdrawal. Compared with a control, this leads successively to an increase in the proliferative cohort, then to a premature depletion of cycling cells. Retinas in which Hh signaling is blocked exhibit opposite shifting phenotypes. $\mathrm{Hh}$ inhibition results in a decreased proliferative pool during early retinogenesis due to slower cell division. However, prolonged precursor proliferation then leads to an increased proportion of cycling cells.

and Xenopus retinas actually decreases in the absence of $\mathrm{Hh}$, along with decreased cell cycle speed. Similarly, $\mathrm{Hh}$ does not favor proliferation versus differentiation per se, as had been suggested by the mouse and chick results, since, along with stimulating S- and M-phase entry, it also brings cells closer to cell cycle withdrawal. Our techniques highlight the fact that the same assay, in this case BrdU incorporation or P-H3 staining, can yield opposite results at different stages, even though the underlying action of the signaling cascade under consideration may be constant.

Our results not only provide a unifying basis for $\mathrm{Hh}$ function in different systems, but also shed light onto previously unexplained findings. For example, a previous study examining early zebrafish retinogenesis found decreased mitotic staining in syu retinas at $34 \mathrm{hpf}$ (Stenkamp et al. 2002), consistent with our interpretation but at odds with the previously proposed $\mathrm{Hh}$ role in zebrafish. Levine et al. (1997) found that addition of recombinant Shh in rodent retinal cell cultures only transiently increases BrdU incorporation and total cell number, which, given our data, likely reflects initial faster cell division followed by premature cell cycle withdrawal. A few previous results, admittedly, are not directly interpretable with our model. Moshiri et al. (2005) did not observe any significant difference in cell cycle duration between control and Shh-treated dissociated retinal precursors in vitro. This may reflect the necessity of preserved intercellular contacts for Hh to act as a regulator of cell cycle speed as previously proposed (Jensen 
and Wallace 1997). Wang et al. (2005) found a precocious birth of RGCs after knocking out Shh in the peripheral mouse retina, whereas we found that RGCs in cyclopamine-treated Xenopus embryos tend to have later birth dates. The discrepancy may arise from the use of different experimental strategies; that is, global inhibition of Hh signaling, which might change the whole environment, versus targeted loss of function in a subpopulation of retinal progenitors. Alternatively, it may reflect a distinct function of Shh in the control of ganglion cell fate (Zhang and Yang 2001; Spence et al. 2004). Clearly, there are likely to be cell-specific and context-dependent effects of Hh signaling in different situations.

We show that $\mathrm{Hh}$ is both necessary and sufficient to induce G1 and G2 acceleration. Recent reports have hinted that this effect may be more general during neural development. Cayuso et al. (2006) found that in the chick spinal cord, Shh inhibition increases the G0/G1 fraction in a mixture of post-mitotic and cycling cells. Our results, from stages when all retinal progenitors are still cycling, suggest that this effect reflects a specific increase in the duration of G1. Additionally, Lien et al. (2006) found that the S-phase fraction in the mouse cerebral cortex increases upon $\mathrm{Hh}$ activation, which in light of our results can be explained by a decreased duration of $\mathrm{G} 1$ and $\mathrm{G} 2$.

These cell cycle accelerating effects of $\mathrm{Hh}$ may be brought about because the expression of key cell cycle stimulators is influenced by $\mathrm{Hh}$ signaling. Cyclin D1 regulates G1/S transition, and, consistent with a slower G1 transition, its expression is decreased upon Hh inhibition, as already reported in the mouse retina (Wang et al. 2005) and other areas of the vertebrate central nervous system (Kenney and Rowitch 2000). The most striking repression by cyclopamine is found for cyclin $B 1$ and $c d c 25 C$, crucial regulators of G2/M transition. These represent key candidates for $\mathrm{Hh}$ effects on G2 length duration. The related $c d c 25 B$ phosphatase was recently found to be a target of Hh signaling in the neural tube (Benazeraf et al. 2006). Finally, expression of cyclin A2, the closest Xenopus homolog to human cyclin $A$, involved in both S- and G2-phase progression /Collins and Garrett 2005), is also significantly reduced upon $\mathrm{Hh}$ blockade. Of note, cyclopamine-treated retinas show decreased expression of the G1-specific cyclin D1 and the G2-specific cyclin B1, despite containing a higher proportion of G1- and G2-phase cells, suggesting that the expression changes are a cause, rather than a consequence, of the proliferation defects. In addition, our short-term gain-of-function analysis is consistent with cyclin D1 being a potential direct target of Hh signaling in the retina, although further investigations will be necessary to address this issue for cyclin A2, cyclin B1, and cdc25. Work in the Drosophila eye has also implicated Hh in the activation of cyclin $D$, cyclin $A$, and $c d c 25$ (string) (Heberlein et al. 1995; Duman-Scheel et al. 2002; Vrailas and Moses 2006). But why does Hh also lead to early cell cycle exit? One possibility is that this effect is not directly dependent on Hh signaling, but is a consequence of the changes in cell cycle kinetics. Alterna- tively, Hh may directly activate the cell cycle exit program through modulation of cyclin-dependent kinase inhibitors expression as proposed in zebrafish (Shkumatava and Neumann 2005).

In the post-embryonic Xenopus retina, the sources of Hh are the ganglion cell layer and the RPE. These regions are juxtaposed to dividing CMZ precursors that presumably cycle fast according to their labeling following a short BrdU pulse and their high expression level of cell cycle activators (Ohnuma et al. 2002a). In contrast, cells in the outermost edge of the CMZ that are not in direct contact with a $\mathrm{Hh}$ source remain BrdU-negative after a short pulse and express low levels of cell cycle activators (Ohnuma et al. 2002a), suggesting slower cell cycle kinetics. Thus, proximal to the source are fast dividing cells almost ready to exit the cell cycle, and distal to the source are slow cycling cells that are far from becoming post-mitotic. It is therefore tempting to speculate that the proliferative properties of CMZ stem cells and precursors are controlled by gradients of Hh signaling. In line with this idea, we have previously shown that the expression of Ptc and Gli1, two known Hh target genes, is detectable in the CMZ region of active proliferation but only faintly detectable in the more peripheral stem cell niche, where Gli3 is highly expressed (Perron et al. 2003). In the absence of Hh signaling, Gli3 is processed into a repressor form (Kasper et al. 2006), which may account for the slower cell cycle kinetics in these most peripheral CMZ stem cells. Consistent with this hypothesis is the recent demonstration that transfection of a repressor form of Gli3 in the chick neural tube causes a severe decrease of proliferation and an increase in the G0/G1-phase fraction (Cayuso et al. 2006). We therefore propose that $\mathrm{Hh}$ signaling transforms retinal stem cells to faster cycling progenitor cells. Some familial and sporadic brain cancers have been shown to derive from inappropriate sustained activation of the $\mathrm{Hh}$ pathway in stem cells or progenitors (Varga et al. 2001; Palma and Ruiz i Altaba 2004). Our work suggests that Hh signaling may contribute to tumor initiation and progression by transforming quiescent or slow cycling cancer stem cells into fast dividing amplifying cells.

\section{Materials and methods}

\section{Embryos and cyclopamine treatment}

$X$. laevis embryos were obtained by hormone-induced egg laying and in vitro fertilization by conventional methods. Cyclopamine treatment (100 $\mu \mathrm{M}_{\text {; }}$ Tebu Bio and TRC) was started from the blastula stage except in Supplementary Figure S2 (from stage 24) and performed as previously described (Perron et al. 2003).

$\mathrm{Smu}^{\mathrm{b641}}$ zebrafish mutants (Varga et al. 2001) were obtained by crossing heterozygous mutants raised at $28.5^{\circ} \mathrm{C}$ and selected according to their external phenotype. Experiments were carried out with wild-type and heterozygous siblings as controls.

\section{Constructs}

The full-length coding region of $X$-bhh was subcloned from the X-bhh T7TS plasmid (Ekker et al. 1995) cut with Spe1, and 
inserted into a pCS2 vector (a gift from D. Turner) following Xbal linearization. The coding region of CD2 (a gift from $\mathrm{N}$. Brown) lacking the signal sequence was subcloned into pCS2 vector cut with Cla1 and Xhol, after PCR amplification. The $\mathrm{N}$-terminal region (devoid of C-terminal cleavage product) of $X$-bhh was subcloned into a pCS2-CD2 vector cut with BamH1 and Cla1, after PCR amplification. The oligonucleotides used are available upon request. The pSP64T VP16GlilzfdGR construct, encoding a glucocortocoid-inducible Glil chimeric morphant, has been described previously (Takabatake et al. 2002). The VP16GlilzfdGR coding region was cut from the pSP64T vector using HindIII/KpnI and was subcloned into a pCS2 vector cut with HindIII/Asp718. The construct is induced with $4 \mu \mathrm{g} /$ $\mathrm{mL}$ DEX (Sigma) in the embryo medium.

\section{In vitro RNA synthesis and microinjections}

Capped GFP and X-Shh (Ekker et al. 1995) RNAs were prepared from CS2 plasmids after NotI digestion, and VP16GlilzfdGR RNAs from a pSP64T plasmid after Kpn1 digestion. Transcription was performed using the mMessage mMachine kit (Ambion). RNAs were injected in a volume of $5 \mathrm{~nL}$ at a concentration of $100-150 \mathrm{pg} / \mathrm{nL}$ into two of two or one of two blastomeres of embryos at the two-cell stage. GFP RNA (100 pg) coinjection was used to visualize injected cells. Morpholino (Mo) injections were performed as indicated by the manufacturer (Genetools). The following antisense Mo oligonucleotide against X-Smo was designed (sequence complementary to $\mathrm{AUG}$ is underlined): CATGGAAGACATCTTGAGAAGTGA. A standard Mo was used as a control (Genetools).

\section{In vivo lipofection}

pCS2-GFP, $p C S 2-X-B h h-C D 2$, and pCS2-VP16GlilzfdGR were transfected at stage 18 into the presumptive region of the retina as previously described (Holt et al. 1990; Ohnuma et al. 2002b).

\section{Brdu incorporation}

BrdU (10 mM; Roche) was injected intra-abdominally for various durations, as indicated in the text. For birthdating analysis, embryos were lipofected at stage 18 and injected every 6-10 h from stage 32 to stage 41 .

\section{Flow cytometry}

Fifty dissected retinas per condition were pooled, transferred to serum-free medium on ice, resuspended either in $\mathrm{Ca}^{2+}$-free medium for $30 \mathrm{~min}$ or in Trypsin-EDTA (Invitrogen) for $15 \mathrm{~min}$, mechanically dissociated into single-cell suspensions, and fixed in ice-cold $70 \%$ ethanol. For DNA content analysis, cells were stained with $40 \mu \mathrm{g} / \mathrm{mL}$ Propidium Iodide (PI; Sigma) in phosphate-buffered saline containing $10 \mu \mathrm{g} / \mathrm{mL}$ RNase. For the BrdU/DNA or phospho-histone H3 (P-H3)/DNA double stainings, single-cell suspensions were subjected to immunodetection as described for slides, before incubation with PI. Cell suspensions were analyzed on a FACScan II (Becton-Dickinson) or a CyAn ADP (Dako), and data were acquired using CellQuest (BD Biosciences) and Summit (Dako).

Data were analyzed with FlowJo (Tree Star), and DNA histograms were deconvolved with MultiCycle (Phoenix Flow Systems). Statistical analysis of overall levels of BrdU incorporation was performed as previously described (Roederer et al. 2001) and with the binomial test. To assess staining and measurement variability, we either stained identically treated samples in parallel, or split one of the samples and analyzed it twice, and compared the distributions, which were not significantly different. For analysis of the BrdU-DNA bivariate graphs, cells were split into the following six categories, according to their DNA content and fluorescence intensity level: (1) S-phase cells ( $S$, BrdU-positive); (2) G2/M cells BrdU-negative $\left(G 2 / M^{-}\right)$; (3) G2/M cells BrdU-positive $\left(G 2 / M^{+}\right)$; (4) G2/M DNA content cells displaying low levels of BrdU incorporation ( $S / G 2$, cells that exited $S$ phase in a small time window just after BrdU injection); (5) G1 cells BrdU-negative $\left(G 1^{-}\right)$; (6) G1/early S-phase DNA content cells displaying low levels of BrdU incorporation (G1/S, cells that entered $S$ phase in a small time window before fixation). The category of G1 BrdU-positive cells was not considered since the duration of the BrdU pulse was less than the G2 + M duration (estimated at $\sim 2 \mathrm{~h}$ for stage 27 , based on our own results). Cells with low levels of BrdU incorporation were defined from the graphs as cells that lie between the clusters of BrdU-positive and BrdU-negative cells. The following four parameters of cell cycle progression were considered: (1) $G 2 M^{-}$cells-that is, G2/M DNA content cells that remain BrdU-negative-were already in $\mathrm{G} 2$ or $M$ phase at the beginning of the BrdU pulse. Therefore, the ratio $G 2 M^{-}:(\operatorname{total} G 2 / M)$, is proportional to the length of $\mathrm{G} 2 / \mathrm{M}, \pi G 2 M^{-}$. Total $G 2 / M$ is calculated as the sum of the $G 2 M^{+}, G 2 M^{-}$, and $S / G 2$ gates. (2) $G 1 / S$ cells have moved from $G 1$ to $S$ during a short interval; hence the ratio $G 1 / S$ : (total G1) can be taken as a rate of G1/S transition, $\Delta G 1$. (3) Similarly, the ratio $S / G 2:($ total $S)$ is a measure of the rate of $S$-phase exit, $\Delta S$. (4) As S-phase cells enter G2, the ratio of $G 2 M^{+}$:(total BrdUpositive), $\pi+G 2 M$, will again reflect the rate of S-phase transition. In the case of experiments done at stage 32 , when some retinal cells have exited the cell cycle, the fraction of cells in G0 was estimated for each condition from the corresponding birthdating experiments and subtracted from the $G 1^{-}$to estimate the G1 proportion. Most experiments (starting from treatment to cell dissociation and analysis) were performed in triplicate. The $\chi^{2}$ test was used to test significance between distributions. To calculate standard errors and confidence intervals, and to assess whether each category was significantly different from the corresponding control category, we assumed a multinomial distribution for cells and used the appropriate binomial test methods (Agresti 1990).

\section{Immunohistochemistry and TUNEL assay}

Immunohistochemistry was performed as described previously (Perron et al. 2003), using mouse monoclonal anti-BrdU (clone BMC 9318; Roche), rabbit polyclonal anti-GFP (Molecular Probe), rabbit polyclonal anti-phospho-histone H3 (Upstate Biotechnology), and anti-mouse or anti-rabbit fluorescent secondary antibodies (Alexa, Molecular Probes). Cell nuclei were counterstained with Hoechst (Sigma). Detection of cell apoptosis was carried out with the DeadEnd fluorometric TUNEL system (Promega). Figures show representative data from one experiment that has been performed at least in duplicate.

\section{In situ hybridization}

Digoxigenin-labeled antisense RNA probes were generated according to the manufacturer's instructions (Roche). Wholemount in situ hybridization was carried out as previously described (Perron et al. 2003). Embryos were then vibratome sectioned $(50 \mu \mathrm{m})$.

\section{Acknowledgments}

We thank S. Ekker, N. Brown, A. Philpott, S. Ohnuma, A. Karaiskou, and K. Takeshima for plasmids. We also thank A. Phil- 
pott and K. Moses for critical reading of the manuscript. We are grateful to N. Miller for instruction in flow cytometry and to G. Lupo for helpful discussions. We thank J. Hamdache for technical assistance. This work was supported by the Wellcome Trust (to W.A.H.) and by an EC Biotechnology grant (QLG3-CT200101460), Retina France, ARC and ANR (to M.P.).

\section{References}

Agresti, A. 1990. Categorical data analysis. Wiley, New York. Ahn, S. and Joyner, A.L. 2005. In vivo analysis of quiescent adult neural stem cells responding to Sonic hedgehog. Nature 437: 894-897.

Amato, M.A., Boy, S., and Perron, M. 2004. Hedgehog signaling in vertebrate eye development: A growing puzzle. Cell. Mol. Life Sci. 61: 899-910.

Benazeraf, B., Chen, Q., Peco, E., Lobjois, V., Medevielle, F., Ducommun, B., and Pituello, F. 2006. Identification of an unexpected link between the Shh pathway and a G2/M regulator, the phosphatase CDC25B. Dev. Biol. 294: 133-147.

Brown, M.H., Krissansen, G.W., Totty, N.F., Sewell, W.A., and Crumpton, M.J. 1987. Purification and N-terminal amino acid sequence of the human T lymphocyte CD2 (T11) surface antigen. Eur. J. Immunol. 17: 15-20.

Cayuso, J., Ulloa, F., Cox, B., Briscoe, J., and Marti, E. 2006. The Sonic hedgehog pathway independently controls the patterning, proliferation and survival of neuroepithelial cells by regulating Gli activity. Development 133: 517-528.

Collins, I. and Garrett, M.D. 2005. Targeting the cell division cycle in cancer: CDK and cell cycle checkpoint kinase inhibitors. Curr. Opin. Pharmacol. 5: 366-373.

Cooper, M.K., Porter, J.A., Young, K.E., and Beachy, P.A. 1998. Teratogen-mediated inhibition of target tissue response to Shh signaling. Science 280: 1603-1607.

Das, A.V., Zhao, X., James, J., Kim, M., Cowan, K.H., and Ahmad, I. 2006. Neural stem cells in the adult ciliary epithelium express GFAP and are regulated by Wnt signaling. Biochem. Biophys. Res. Commun. 339: 708-716.

Dolbeare, F., Gratzner, H., Pallavicini, M.G., and Gray, J.W. 1983. Flow cytometric measurement of total DNA content and incorporated bromodeoxyuridine. Proc. Natl. Acad. Sci. 80: $5573-5577$.

Dorsky, R.I., Rapaport, D.H., and Harris, W.A. 1995. Xotch inhibits cell differentiation in the Xenopus retina. Neuron 14: 487-496.

Duman-Scheel, M., Weng, L., Xin, S., and Du, W. 2002. Hedgehog regulates cell growth and proliferation by inducing Cyclin D and Cyclin E. Nature 417: 299-304.

Ekker, S.C., McGrew, L.L., Lai, C.J., Lee, J.J., von Kessler, D.P., Moon, R.T., and Beachy, P.A. 1995. Distinct expression and shared activities of members of the Hedgehog gene family of Xenopus laevis. Development 121: 2337-2347.

Harris, W.A. and Perron, M. 1998. Molecular recapitulation: The growth of the vertebrate retina. Int. J. Dev. Biol. 42: 299-304.

Heberlein, U., Singh, C.M., Luk, A.Y., and Donohoe, T.J. 1995. Growth and differentiation in the Drosophila eye coordinated by hedgehog. Nature 373: 709-711.

Holt, C.E., Bertsch, T.W., Ellis, H.M., and Harris, W.A. 1988. Cellular determination in the Xenopus retina is independent of lineage and birth date. Neuron 1: 15-26.

Holt, C.E., Garlick, N., and Cornel, E. 1990. Lipofection of cDNAs in the embryonic vertebrate central nervous system. Neuron 4: 203-214.

Incardona, J.P., Gaffield, W., Kapur, R.P., and Roelink, H. 1998.
The teratogenic Veratrum alkaloid cyclopamine inhibits sonic hedgehog signal transduction. Development 125: 3553-3562.

Inoue, T., Kagawa, T., Fukushima, M., Shimizu, T., Yoshinaga, Y., Takada, S., Tanihara, H., and Taga, T. 2006. Activation of canonical Wnt pathway promotes proliferation of retinal stem cells derived from adult mouse ciliary margin. Stem Cells 24: 95-104.

Jensen, A.M. and Wallace, V.A. 1997. Expression of Sonic hedgehog and its putative role as a precursor cell mitogen in the developing mouse retina. Development 124: 363-371.

Kasper, M., Regl, G., Frischauf, A.M., and Aberger, F. 2006. GLI transcription factors: Mediators of oncogenic Hedgehog signaling. Eur. J. Cancer 42: 437-445.

Kenney, A.M. and Rowitch, D.H. 2000. Sonic hedgehog promotes $G(1)$ cyclin expression and sustained cell cycle progression in mammalian neuronal precursors. Mol. Cell. Biol. 20: 9055-9067.

Kubo, F., Takeichi, M., and Nakagawa, S. 2003. Wnt 2 b controls retinal cell differentiation at the ciliary marginal zone. Development 130: 587-598.

Lai, K., Kaspar, B.K., Gage, F.H., and Schaffer, D.V. 2003. Sonic hedgehog regulates adult neural progenitor proliferation in vitro and in vivo. Nat. Neurosci. 6: 21-27.

Levine, E.M., Roelink, H., Turner, J., and Reh, T.A. 1997. Sonic hedgehog promotes rod photoreceptor differentiation in mammalian retinal cells in vitro. J. Neurosci. 17: 6277-6288.

Lien, W.H., Klezovitch, O., Fernandez, T.E., Delrow, J., and Vasioukhin, V. 2006. $\alpha \mathrm{E}$-Catenin controls cerebral cortical size by regulating the hedgehog signaling pathway. Science 311: 1609-1612.

Lupo, G., Liu, Y., Qiu, R., Chandraratna, R.A., Barsacchi, G., He, R.Q., and Harris, W.A. 2005. Dorsoventral patterning of the Xenopus eye: A collaboration of Retinoid, Hedgehog and FGF receptor signaling. Development 132: 1737-1748.

Machold, R., Hayashi, S., Rutlin, M., Muzumdar, M.D., Nery, S., Corbin, J.G., Gritli-Linde, A., Dellovade, T., Porter, J.A., Rubin, L.L., et al. 2003. Sonic hedgehog is required for progenitor cell maintenance in telencephalic stem cell niches. Neuron 39: 937-950.

Masai, I., Yamaguchi, M., Tonou-Fujimori, N., Komori, A., and Okamoto, H. 2005. The hedgehog-PKA pathway regulates two distinct steps of the differentiation of retinal ganglion cells: The cell-cycle exit of retinoblasts and their neuronal maturation. Development 132: 1539-1553.

Moshiri, A. and Reh, T.A. 2004. Persistent progenitors at the retinal margin of $\mathrm{ptc}^{+/-}$mice. J. Neurosci. 24: 229-237.

Moshiri, A., McGuire, C.R., and Reh, T.A. 2005. Sonic hedgehog regulates proliferation of the retinal ciliary marginal zone in posthatch chicks. Dev. Dyn. 233: 66-75.

Neumann, C.J. and Nuesslein-Volhard, C. 2000. Patterning of the zebrafish retina by a wave of sonic hedgehog activity. Science 289: 2137-2139.

Nowakowski, R.S., Lewin, S.B., and Miller, M.W. 1989. Bromodeoxyuridine immunohistochemical determination of the lengths of the cell cycle and the DNA-synthetic phase for an anatomically defined population. J. Neurocytol. 18: 311318.

Ohnuma, S., Hopper, S., Wang, K.C., Philpott, A., and Harris, W.A. 2002a. Co-ordinating retinal histogenesis: Early cell cycle exit enhances early cell fate determination in the Xenopus retina. Development 129: 2435-2446.

Ohnuma, S., Mann, F., Boy, S., Perron, M., and Harris, W.A. 2002b. Lipofection strategy for the study of Xenopus retinal development. Methods 28: 411-419.

Palma, V. and Ruiz i Altaba, A. 2004. Hedgehog-GLI signaling 
Locker et al.

regulates the behavior of cells with stem cell properties in the developing neocortex. Development 131: 337-345.

Palma, V., Lim, D.A., Dahmane, N., Sanchez, P., Brionne, T.C., Herzberg, C.D., Gitton, Y., Carleton, A., Alvarez-Buylla, A., and Ruiz i Altaba, A. 2005. Sonic hedgehog controls stem cell behavior in the postnatal and adult brain. Development 132: 335-344.

Perron, M., Kanekar, S., Vetter, M.L., and Harris, W.A. 1998. The genetic sequence of retinal development in the ciliary margin of the Xenopus eye. Dev. Biol. 199: 185-200.

Perron, M., Boy, S., Amato, M.A., Viczian, A., Koebernick, K., Pieler, T., and Harris, W.A. 2003. A novel function for Hedgehog signaling in retinal pigment epithelium differentiation. Development 130: 1565-1577.

Quastler, H. and Sherman, F.G. 1959. Cell population kinetics in the intestinal epithelium of the mouse. Exp. Cell Res. 17: 420-438.

Roederer, M., Treister, A., Moore, W., and Herzenberg, L.A. 2001. Probability binning comparison: A metric for quantitating univariate distribution differences. Cytometry 45: 3746.

Roy, S. and Ingham, P.W. 2002. Hedgehogs tryst with the cell cycle. J. Cell Sci. 115: 4393-4397.

Shkumatava, A. and Neumann, C.J. 2005. Shh directs cell-cycle exit by activating p57Kip2 in the zebrafish retina. $E M B O$ Rep. 6: 563-569.

Spence, J.R., Madhavan, M., Ewing, J.D., Jones, D.K., Lehman, B.M., and Del Rio-Tsonis, K. 2004. The Hedgehog pathway is a modulator of retina regeneration. Development 131: 46074621.

Stadler, J.A., Shkumatava, A., and Neumann, C.J. 2004. The role of hedgehog signaling in the development of the zebrafish visual system. Dev. Neurosci. 26: 346-351.

Stenkamp, D.L., Frey, R.A., Mallory, D.E., and Shupe, E.E. 2002. Embryonic retinal gene expression in sonic-you mutant zebrafish. Dev. Dyn. 225: 344-350.

Takabatake, Y., Takabatake, T., Sasagawa, S., and Takeshima, K. 2002. Conserved expression control and shared activity between cognate $\mathrm{T}$-box genes $\mathrm{Tbx} 2$ and $\mathrm{Tbx} 3$ in connection with Sonic hedgehog signaling during Xenopus eye development. Dev. Growth Differ. 44: 257-271.

Van Raay, T.J., Moore, K.B., Iordanova, I., Steele, M., Jamrich, M., Harris, W.A., and Vetter, M.L. 2005. Frizzled 5 signaling governs the neural potential of progenitors in the developing Xenopus retina. Neuron 46: 23-36.

Varga, Z.M., Amores, A., Lewis, K.E., Yan, Y.L., Postlethwait, J.H., Eisen, J.S., and Westerfield, M. 2001. Zebrafish smoothened functions in ventral neural tube specification and axon tract formation. Development 128: 3497-3509.

Vernon, A.E. and Philpott, A. 2003. The developmental expression of cell cycle regulators in Xenopus laevis. Brain Res. Gene Expr. Patterns 3: 179-192.

Vrailas, A.D. and Moses, K. 2006. Smoothened, thickveins and the genetic control of cell cycle and cell fate in the developing Drosophila eye. Mech. Dev. 123: 151-165.

Wallace, V.A. and Raff, M.C. 1999. A role for Sonic hedgehog in axon-to-astrocyte signaling in the rodent optic nerve. Development 126: 2901-2909.

Wang, Y., Dakubo, G.D., Thurig, S., Mazerolle, C.J., and Wallace, V.A. 2005. Retinal ganglion cell-derived sonic hedgehog locally controls proliferation and the timing of RGC development in the embryonic mouse retina. Development 132: 5103-5113.

Wechsler-Reya, R.J. and Scott, M.P. 1999. Control of neuronal precursor proliferation in the cerebellum by Sonic Hedgehog. Neuron 22: 103-114.
Wetts, R., Serbedzija, G.N., and Fraser, S.E. 1989. Cell lineage analysis reveals multipotent precursors in the ciliary margin of the frog retina. Dev. Biol. 136: 254-263.

Zhang, X.M. and Yang, X.J. 2001. Regulation of retinal ganglion cell production by Sonic hedgehog. Development 128: 943957. 


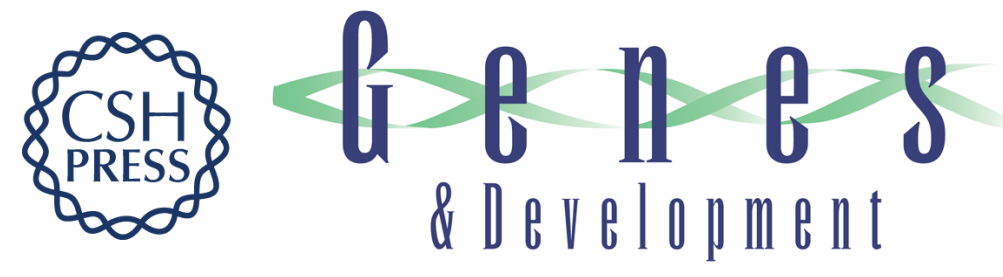

\section{Hedgehog signaling and the retina: insights into the mechanisms controlling the proliferative properties of neural precursors}

Morgane Locker, Michalis Agathocleous, Marcos A. Amato, et al.

Genes Dev. 2006, 20:

Access the most recent version at doi:10.1101/gad.391106

Supplemental http://genesdev.cshlp.org/content/suppl/2006/10/20/20.21.3036.DC1
Material

References This article cites 55 articles, 22 of which can be accessed free at:

http://genesdev.cshlp.org/content/20/21/3036.full.html\#ref-list-1

License

Email Alerting Receive free email alerts when new articles cite this article - sign up in the box at the top

Service right corner of the article or click here.

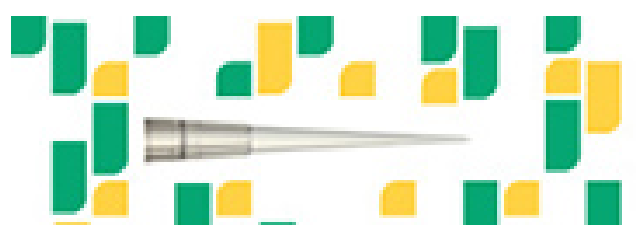

Focused on your science. 